\title{
DISTRIBUCIÓN ESPACIAL DEL RIESGO DE PRECIPITACIONES EXTREMAS EN EL PIRINEO ARAGONÉS OCCIDENTAL
}

\author{
Santiago BEGUERÍA y Adrián LORENTE \\ Instituto Pirenaico de Ecología, CSIC, Campus de Aula Dei, Zaragoza
}

Resumen: Los eventos pluviométricos extremos son episodios lluviosos de gran intensidad capaces de desencadenar procesos geomorfológicos de gran magnitud e importantes consecuencias en pérdidas económicas y humanas, como conocemos por ejemplos recientes e históricos. Estos efectos devastadores son si cabe mayores en áreas de montaña, de gran energía topográfica e inestabilidad geomorfológica. Además de su intensidad, estos episodios se caracterizan por su baja frecuencia temporal y por presentar una distribución espacial aparentemente errática. Este carácter extremo hace que las series pluviométricas normalmente disponibles sean poco adecuadas para el estudio de las precipitaciones máximas.

En este artículo se estudia la distribución espacial del riesgo de precipitaciones extremas en el Pirineo occidental aragonés, buscando posibles patrones geográficos y explorando la posibilidad de realizar una cartografía del riesgo de precipitaciones extremas mediante técnicas estadísticas y SIG. Se revisa la metodología estadística habitual en el estudio de los eventos extremos (ajuste de los datos a una ley de probabilidad y cálculo de períodos de retorno). Este tipo de modelos, muy sensibles a los datos, requieren un estudio de significación estadística para comprobar su fiabilidad.

Palabras clave: Precipitación, Eventos pluviométricos extremos, Períodos de retomo, Riesgos hidrogeomorfológicos, Pirineo aragonés

\begin{abstract}
Extreme rainfall events are very unusual and tend to provoke high magnitude geomorphic processes, with catastrophic economic and human consequences. The hazard is even higher in mountain areas, with huge topographic gradients and high geomorphic instability. These extreme events occur with a low frequency and an apparently erratic spatial distribution. This is the reason why the current pluviometric records are inadequate or insufficient for studying the spatial and temporal organisation of the most intense rainstorms.
\end{abstract}

In this paper the spatial distribution of the extreme rainfalls in the Central Spanish Pyrenees has been assessed. A map of extreme precipitation hazard with uncertainty, using statistical techniques and GIS, is proposed. A critical analysis of the statistical 
methods used in the study of extreme rainfall events is done. Such methods need a study of the statistical significance in order to test their reliability.

Key words: Rainfall, Extreme events, Return periods, Hydrogeomorphological hazards, Central Spanish Pyrenees

\section{INTRODUCCIÓN}

El estudio de los episodios pluviométricos extremos tiene una larga tradición en Hidrología y Geomorfología. Es durante estos eventos cuando tiene lugar una gran parte del trabajo geomorfológico, activándose procesos de erosión superficial, arroyamiento torrencial, movimientos en masa, cambios en los cauces y en las llanuras aluviales, etc. (ver, por ej., DUNNE y LEOPOLD, 1978; GARCÍA RUIZ et al.,1999; GARCÍA RUIZ et al., en prensa $a$ ). En muchas ocasiones estos procesos cobran una dimensión catastrófica, afectando a infraestructuras e incluso cobrándose vidas humanas.

En el ámbito de la península Ibérica son especialmente violentos los episodios ligados a la frontogénesis mediterránea, fundamentalmente otoñal, que afecta de manera generalizada a la franja litoral. Las regiones montañosas constituyen otro ámbito de riesgo, pues la complejidad orográfica favorece el desarrollo de tormentas convectivas de gran intensidad y amplifica el efecto de las perturbaciones frontales al obligar a ascender a las masas de aire. Por otra parte, la gran energía del relieve facilita que estos episodios de precipitación intensa desencadenen procesos geomorfológicos de envergadura, que pueden afectar a los asentamientos humanos al emplazarse éstos en zonas de riesgo como fondos de valle o conos de deyección de torrentes.

En el caso del Pirineo español el registro histórico es rico en ejemplos de tales episodios extremos (VILAR, 1995; MARTÍN VIDE, 1985), con frecuentes precipitaciones superiores a los $100 \mathrm{~mm}$ en $24 \mathrm{~h}$. Algunos casos excepcionales son los $600 \mathrm{~mm}$ registrados en el refugio de Góriz (Ordesa, $2200 \mathrm{~m}$ ), los $500 \mathrm{~mm}$ de Sin, los $307 \mathrm{~mm}$ de Urdiceto, los $252 \mathrm{~mm}$ de Capella, o los 214 de Gistaín en noviembre de 1982 (MARTí BONO y PUIGDEFÁBREGAS, 1983). Otros casos ejemplifican la violencia de los procesos hidrogeomorfológicos asociados a las lluvias extremas, como el desastre del camping de Biescas en agosto de 1996 (GARCÍA RUIZ et al., 1996; WHITE et al., 1997) o las riadas y coladas de bloques en los conos de deyección de los barrancos de Remáscaro y La Guingueta en noviembre de 1982 (BRU et al., 1984; CLOTET et al., 1989).

El presente trabajo pretende contribuir al conocimiento de las precipitaciones extremas en el Pirineo aragonés occidental, determinando la existencia o no de patrones en su distribución espacial. Se hace hincapié en los problemas que conlleva el manejo estadístico de los valores extremos de una distribución, y en la necesidad de acompañar los cálculos de perí- 
odos de retorno del correspondiente análisis de significación. Finalmente, se presenta una cartografía del riesgo de precipitaciones extremas a partir de técnicas estadísticas y SIG.

\section{ÁREA DE ESTUDIO}

El trabajo cubre la parte del Pirineo aragonés y navarro comprendida entre los ríos Esca y Gállego, desde el eje de la cordillera hasta las Sierras Exteriores (Figura 1). Este rectángulo de unos $3.600 \mathrm{~km}^{2}$ recoge gran parte de la variabilidad paisajística del Pirineo central español, desde la alta montaña hasta las depresiones intramontanas. El relieve se ordena en bandas sucesivas de orientación general oeste-este, distinguiéndose de norte a sur los siguientes sectores:

El Alto Pirineo paleozoico o Pirineo axial, con un relieve muy diversificado debido a la heterogeneidad litológica. Las mayores altitudes se alcanzan en los batolitos graníticos

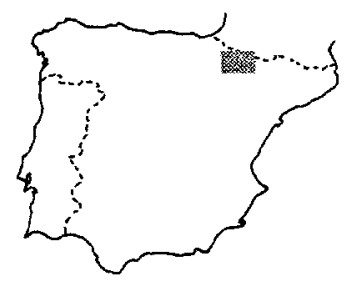

FRANCIA

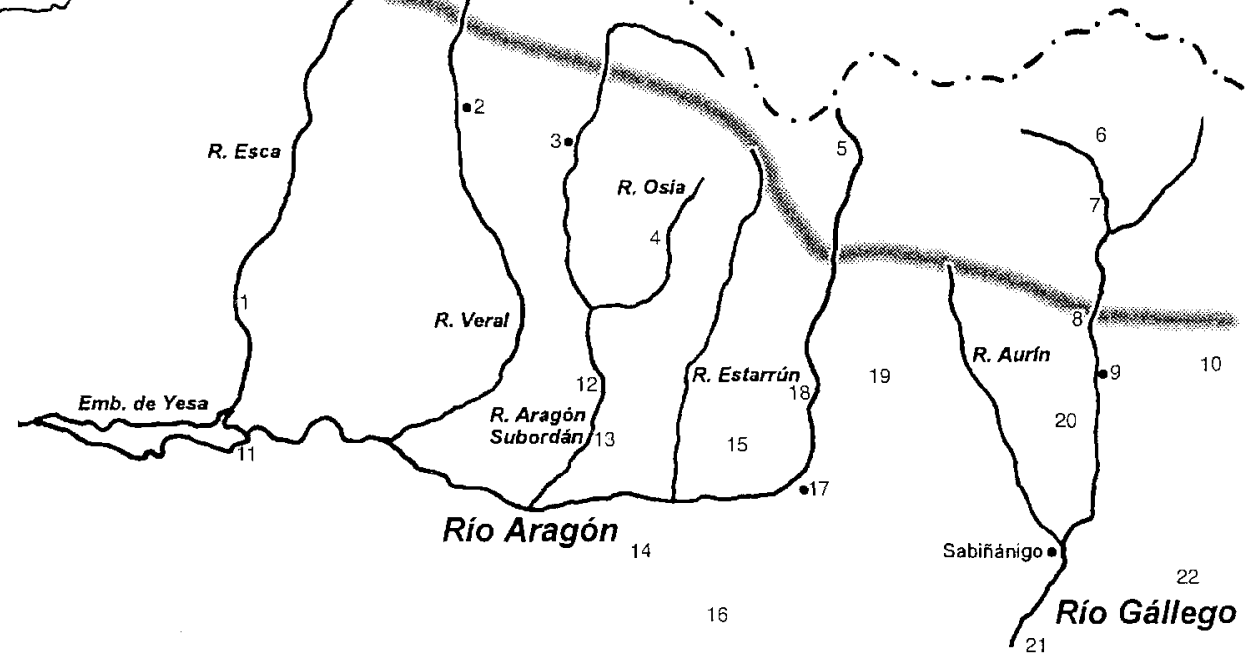

Figura 1. Area de estudio, con indicación del eje de las Sierras Interiores. Los números hacen referencia a las estaciones meteorológicas empleadas en este trabajo. Estaciones del Alto

Pirineo: 1, Salvatierra de Escá; 2, Ansó; 3, Hecho; 4, Aragüés del Puerto; 5, Rioseta; 6, La Sarra; 7, Escarrilla; 8, Búbal; 9, Biescas; 10, Yésero. Resto de estaciones: 11, Artieda; 12, Embún; 13, Javierregay; 14, Binacua; 15, Caniás; 16, Botaya; 17, Jaca; 18, Castiello de Jaca; 19, Bescós de Garcipollera; 20, Escuer; 21, Hostal de Ipiés; 22, Yebra de Basa 
de Panticosa y de Balaitús (3.144 m) o en su orla metamórfica (Infiernos, $3.082 \mathrm{~m}$ ), así como en pitones volcánicos andesíticos (Anayet, $2.559 \mathrm{~m}$ ) y en afloramientos calizos (Peña Foratata, $2.341 \mathrm{~m}$ ). Los dominios pizarrosos (Silúrico) y arcillosos (Pérmico) dan lugar, en cambio, a relieves de pendientes suaves y topografía relativamente deprimida (alto valle de Tena, Tramacastilla, Candanchú-Somport, Selva de Oza).

Las Sierras Interiores, armadas en calizas y areniscas mesozoicas forman una alineación notablemente continua y de relieve muy abrupto: Peña Forca $(2.390 \mathrm{~m})$, Bisaurín $(2.668 \mathrm{~m})$, Aspe $(2.645 \mathrm{~m})$, Collarada $(2.886 \mathrm{~m})$, Telera $(2.769 \mathrm{~m})$, Tendeñera $(2.853 \mathrm{~m})$ (Figura 1$)$.

Al sur de las Sierras Interiores la altitud desciende gradualmente en el sector del flysch eoceno, con un relieve dominado por divisorias suaves y laderas regularizadas cuyas cotas máximas apenas superan los $2.000 \mathrm{~m}$.

La Depresión Interior Altoaragonesa se modela en margas de edad eocena, dando lugar a un amplio corredor (Canal de Berdún y Val Ancha) con relieve de glacis y terrazas aluviales cuyo fondo oscila entre 600 y $850 \mathrm{~m}$.

Finalmente, el sector más meridional corresponde al Prepirineo, formado por areniscas y conglomerados suavemente plegados (peñas de San Juan y Oroel, 1.769 m; depresión Guarguera-Onsella), y por las Sierras Exteriores, que reproducen en pequeño las características de las Sierras Interiores. Culminan en el Tozal de Guara (2.077 m).

La disposición general de los valles es N-S, con las excepciones del río Aragón en la Depresión Interior y de algunos afluentes menores.

El clima es de tipo montano, dominado por las influencias oceánicas al noroeste y mediterráneas hacia el sur y sudeste (CREUS NOVAU, 1983). La oceanidad es patente al norte de las Sierras Interiores, donde las precipitaciones tienden a concentrarse en la estación fría, con registros medios anuales superiores a $1200 \mathrm{~mm}$.

Las Sierras Interiores ejercen un efecto de pantalla orográfica, dejando a los territorios situados al sur en condiciones de sombra pluviométrica con respecto a los flujos zonales. En general, hacia el sur y el sudeste el clima adquiere un carácter de tipo submediterráneo con matices continentales. Las precipitaciones anuales se cifran entre 700 y $1.000 \mathrm{~mm}$, con máximos equinocciales, aunque se superan esos valores en los relieves más prominentes.

\section{MÉTODOS}

En Hidrología los riesgos suelen establecerse a partir de los llamados períodos de retorno o intervalos de recurrencia, que no son sino la inversa de la probabilidad: a un evento con 
una probabilidad de ocurrencia en un año de 1/100 le corresponde un período de retorno de 100 años. La aproximación habitual al problema es, pues, estadístico-frecuentista: a partir de los registros de precipitación existentes se hacen las estimaciones de los períodos de retorno. Normalmente se toma la máxima precipitación en 24 horas de cada año, lo cual asegura la independencia de los sucesos, y la serie resultante se ajusta a una distribución de probabilidad de valores máximos: log-Normal, log-Pearson III, Gumbel-tipo I, Weibulltipo III, Gamma (CHOW, 1994; LLAMAS, 1993; JONES, 1997; CUSTODIO y LLAMAS, 1996; APARICIO, 1997).

Una vez determinada la ley de probabilidad se pueden realizar extrapolaciones y calcular los períodos de retorno de cualquier precipitación mediante la siguiente relación:

$$
\operatorname{Tr}_{x}=\frac{1}{1-p_{x}}=\frac{1}{q_{x}}
$$

donde:

$\operatorname{Tr}_{x}$ es el tiempo de retorno de una precipitación de $x \mathrm{~mm}$, en años;

$p_{x}$ es la probabilidad de ocurrencia anual de un evento inferior a $\mathrm{x}$ mm;

$q_{x}$ es la probabilidad de ocurrencia anual de un evento igual o superior a $\mathrm{xmm}$.

Para este estudio se han recopilado los registros diarios de precipitación de 22 estaciones meteorológicas pertenecientes a las redes del Instituto Nacional de Meteorología y del Instituto Pirenaico de Ecología, con longitudes de 15 a 25 años (Figura 1). Se ha extraído la serie de precipitaciones máximas anuales de cada estación, y dichas series se han ajustado a una distribución asintótica de Gumbel-tipo I, cuya función de distribución es la siguiente (GUMBEL, 1962):

$$
F_{(x)}=e^{-e^{-y}}=p_{x}
$$

siendo:

$F_{(x)}$ la probabilidad de que en un año se registre una precipitación en $24 \mathrm{~h}$. inferior a $\mathrm{xm}$. $\mathrm{F}_{(\mathrm{x})}=\mathrm{p}=\mathrm{P}(\mathrm{x}<\mathrm{X})$;

e la base de los logaritmos neperianos;

y la variable reducida, calculada a partir de la expresión:

donde:

$$
y=\alpha(x-\beta)
$$

$$
-\infty<x<\infty
$$


$\alpha$ y $\beta$ son parámetros de la distribución, de forma y escala respectivamente. Se han estimado a partir de la media aritmética y la desviación estándar de la muestra (CHOW, 1994:397; LLAMAS, 1993:136-137; CUSTODIO y LLAMAS, 1996:149. Otros autores proponen métodos ligeramente diferentes: ver también APARICIO, 1997:264):

$$
\begin{gathered}
\alpha=\frac{1.2825}{S_{x}} \\
\beta=\bar{x}-\frac{0.5772}{\alpha}=\bar{x}-045 S_{x} .
\end{gathered}
$$

Todas las técnicas de inferencia estadística (estimación de los parámetros de una población a partir de una muestra) son sensibles a la información de partida. Este hecho hace especialmente delicado trabajar con los datos extremos de una muestra, como sucede con las precipitaciones máximas, pues evidentemente se encuentran mucho peor representados en la misma que los valores cercanos al promedio. Como se ha observado en anteriores trabajos (WHITE et al., 1997), es perfectamente posible que en un registro de 20 años no esté representado el evento correspondiente a un período de retorno de 20 años, con lo que los resultados del modelo no serán correctos. Lo mismo sucedería si en esos 20 años se hubiera registrado el evento correspondiente a un retorno de 100 años. Es necesario, pues, realizar un estudio sobre el error que cabe esperar en las estimaciones inferidas.

Se ha calculado para cada curva de períodos de retorno la banda de confianza a un nivel de significación 90\%. Si se pudiera disponer del registro infinito de todas las precipitaciones caídas en una localidad, y se extrajera del mismo un número suficientemente grande de muestras de tamaños iguales, se puede demostrar que alrededor del $65 \%$ de las probabilidades y períodos de retorno calculados a partir de dichas muestras estarían dentro de una banda de $\sigma_{x}$ (error estándar); $y$, en general, dentro de una banda de $z \sigma_{x^{\prime}}$ siendo $z$ el valor de la variable normal estandarizada correspondiente al nivel de significación escogido (1,645 para un nivel de significación 90\%). La fórmula del error estándar de una distribución de Gumbel-tipo I es la siguiente:

$$
\sigma_{x}=\frac{\sqrt{p_{x} \cdot q_{x}}}{\sqrt{n} \cdot e^{-y-e^{-y}}}
$$

donde $n$ es el número de registros de la serie.

Para calcular los límites superior e inferior de la banda de confianza $\left(F S_{(x),}, F i_{(x)}\right)$ aplicaremos la fórmula [2] a los valores de $x$ modificados por el error al nivel de significación escogido:

$$
\begin{aligned}
& F S_{(x)}=F_{\left(x+z \sigma_{x}\right)} \\
& F i_{(x)}=F_{\left(x-z \sigma_{x}\right)}
\end{aligned}
$$


Se ha escogido la precipitación máxima de 100 años de retorno $\left(P_{100}\right.$ en adelante $)$ como indicador del riesgo de ocurrencia de precipitaciones extremas en un punto. Se ha llevado a cabo un análisis de regresión entre $P_{100}$ y una serie de variables climáticas, topográficas y de posición, partiendo de la hipótesis de que el relieve local es determinante en la distribución de lluvias extremas en zonas de montaña. Las variables regresoras, extraídas en su mayor parte de un modelo digital de elevaciones (GTOPO-30, United States Geological Survey), son las siguientes:

- Localización geográfica de la estación: coordenadas UTM (dos variables: easting, northing).

- Altitud de la estación.

- Aspecto u orientación de la vertiente donde está instalada la estación.

- Altitud máxima en círculos de 1,3,5 y $8 \mathrm{~km}$ en torno a la estación (cuatro variables).

- Altitud mínima en círculos de 1,3,5 y $8 \mathrm{~km}$ en torno a la estación (cuatro variables).

- Gradiente topográfico en un círculo de 1, 3, 5 y $8 \mathrm{~km}$ en torno a la estación (tres variables).

- Desviación estándar de las altitudes en círculos de 1,3,5 y $8 \mathrm{~km}$ en torno a la estación, como indicador de la heterogeneidad del relieve (cuatro variables).

- Promedio y desviación típica de las precipitaciones anuales (dos variables).

Se han calculado los coeficientes de correlación $r$ de Pearson entre $\mathrm{P}_{100}$ y cada una de las variables regresoras. Considerándose que una combinación de varias de ellas podía ofrecer mejores resultados, se ha utilizado el paquete estadístico Statgraphics v. 2.1 que ensayó modelos de regresión múltiple con todas las combinaciones posibles de las variables, llegando a un modelo óptimo de nueve parámetros. Por último se introdujo este modelo paramétrico en un sistema de información geográfica (IDRISI for Windows v. 2.0) para la elaboración del mapa de riesgos de precipitaciones extremas.

\section{RESULTADOS Y DISCUSIÓN}

\section{Precisión e incertidumbre en el cálculo de períodos de retorno}

En la Tabla 1 se muestran las series de precipitaciones máximas anuales y las precipitaciones máximas calculadas para diferentes períodos de retorno. La precipitación máxima 

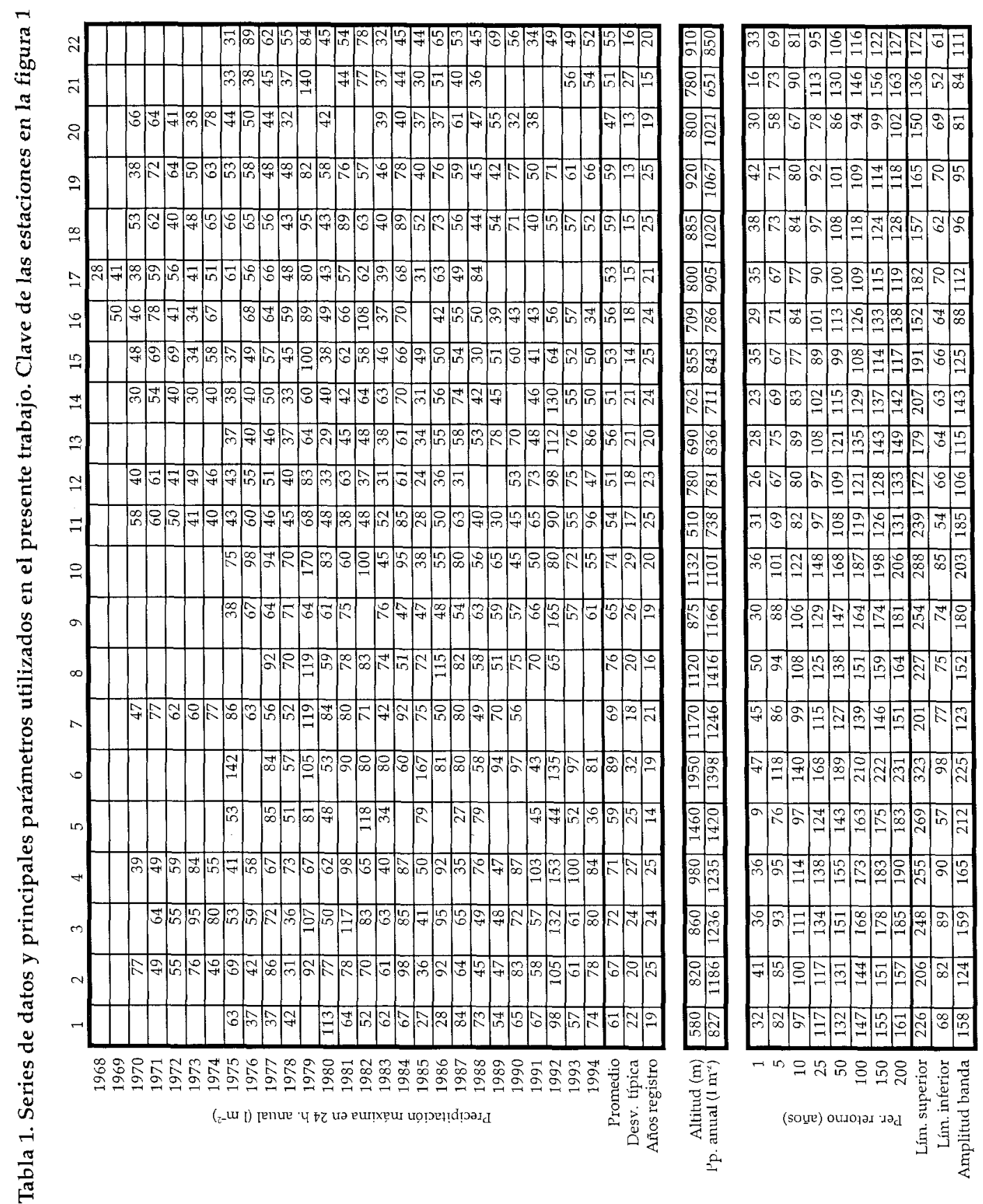
anual oscila, en la mayoría de las estaciones estudiadas, entre 50 y $75 \mathrm{~mm}$. La irregularidad interanual es notable (de un cuarto a un tercio sobre el promedio). En el conjunto de las estaciones estudiadas se han registrado un total de 24 eventos pluviométricos con intensidades diarias superiores a los $100 \mathrm{~mm}$, destacando los de Yésero (170 $\mathrm{mm}$ en 1979), La Sarra (167 mm en 1985), Biescas (165 mm en 1992) o Aragüiés (153 mm en 1992). Eventos de mayor intensidad han sido registrados en otras localidades pirenaicas (ver introducción), debiéndose considerar como casos extremos entre los extremos.

En la Tabla 1 se han consignado también las precipitaciones máximas calculadas para recurrencias de 1, 5, 10, 25, 50, 100, 150 y 200 años. En la mayoría de los casos los eventos estimados se sitúan entre 70 y $100 \mathrm{~mm}$ para 5 años de recurrencia, 100 y $140 \mathrm{~mm}$ para 25 años, o 110 y $180 \mathrm{~mm}$ para 100 años. Esta información suele representarse en una gráfica semilogarítmica que relaciona el período de retorno/probabilidad anual con el evento que le corresponde. En la Figura 2A/B se muestran los ejemplos contrastados de La Sarra (alto valle del Gállego) y Jaca (Depresión Interior). Los puntos negros son los eventos registrados en la serie, y la línea continua representa el modelo teórico, cuya predicción podemos extender más allá de la longitud del registro inicial. La precipitación máxima esperable aumenta con el período de retorno, aunque la tasa de variación disminuye exponencialmente con el tiempo: en La Sarra (Figura 2A), el evento de 1 año de recurrencia ( $40 \mathrm{~mm}$ $\mathrm{dia}^{-1}$ ) se puede duplicar en 3 años, mientras que el evento de 5 años de recurrencia (120 $\mathrm{mm} \mathrm{dia}^{-1}$ ) no se duplicará hasta más de 200 años después. La pendiente de la recta permite comparar diferentes estaciones, como por ejemplo Jaca (Figura 2A), que presenta una escasa pendiente y por tanto un bajo nivel de riesgo de precipitaciones extremas, y La Sarra (Figura 2B), en la que la torrencialidad de las precipitaciones es mucho mayor.

Sin embargo, hay que tener en cuenta que cuando estimamos un parámetro a partir de una muestra (como al calcular períodos de retorno) sólo escogemos el valor más probable dentro de su propia distribución de probabilidad, con lo que la probabilidad de acertar es del $50 \%$. La banda de confianza nos da el rango de valores dentro del cual se encontrará la variable que tratamos de estimar, con un grado de certeza (nivel de significación) conocido. En la Figura 2C se muestra la gráfica de períodos de retorno para la estación de Jaca, a la que se ha añadido la banda de confianza al nivel de significación $90 \%$. Al calcular períodos de retorno, el error es mayor cuanto más lejana sea la prospección. En la figura, por ejemplo, se observa cómo es mucho más imprecisa la estimación del evento de 500 años de retorno $(>50,120,<185)$ que la del de $100(>70,110,<180)$.

Por supuesto, la ganancia en fiabilidad o confianza supone una pérdida proporcional de precisión, pues la anchura de la banda se incrementa con el nivel de significación. En la Figura 2D se puede ver cómo varía la banda de confianza al variar todos estos parámetros, siendo: $a$ la línea de máxima probabilidad $(50 \%) ; b_{1}$ y $b_{2}$ los límites de la banda de confianza al nivel de significación $90 \%$, a partir de una serie de 20 años de longitud; $c_{1}$ y $c_{2}$ los mismos límites pero a partir de un registro de 100 años de duración. Esta última banda equivale aproximadamente a trabajar al 70\% de significación con la serie inicial de 20 años de longitud. 

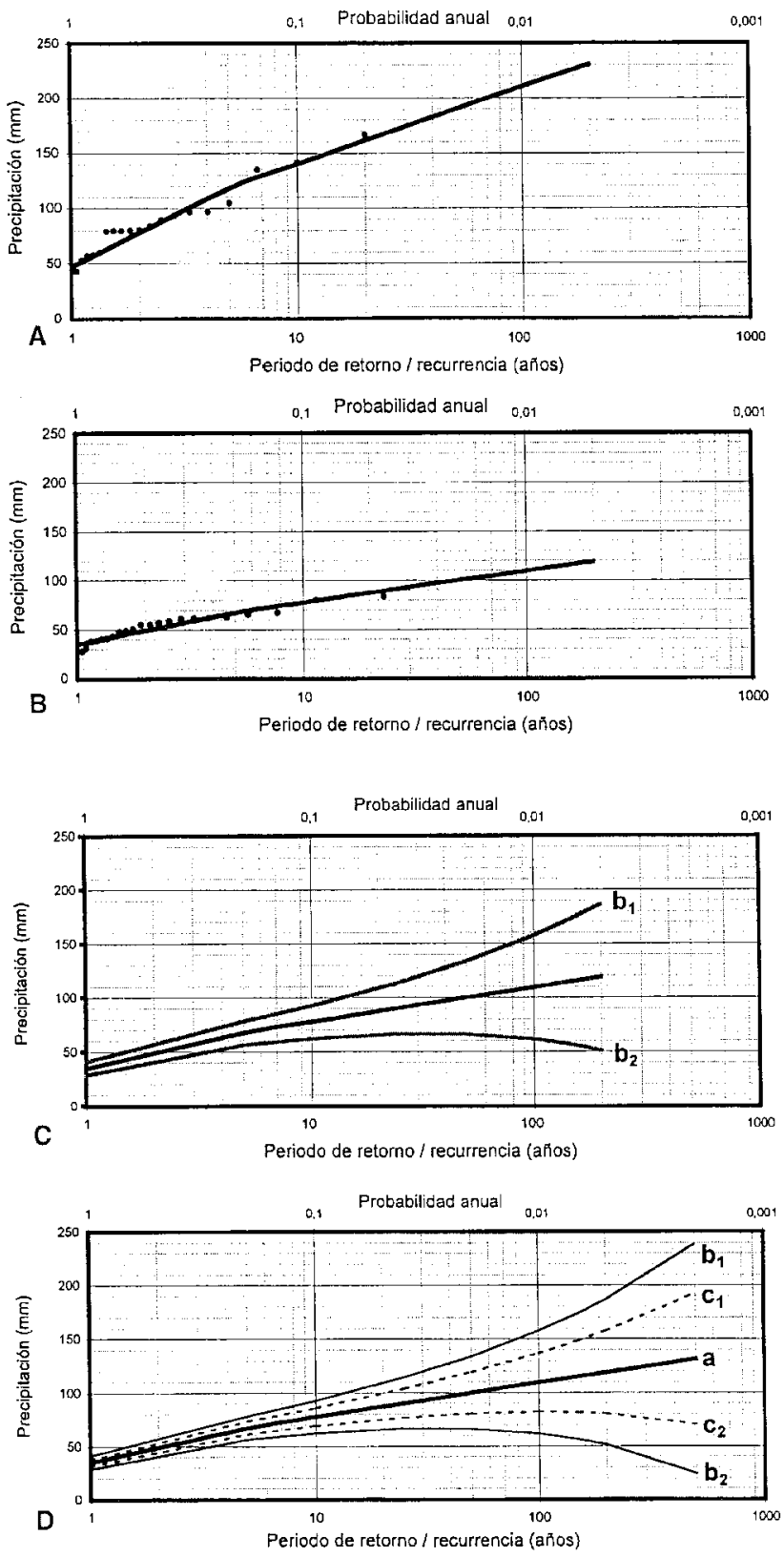

Figura 2. Precipitación correspondiente a diferentes períodos de retorno. A.

Estación meteorológica de La Sarra (alto Gállego). B. Estación meteorológica de Jaca. C.

Estación meteorológica de Jaca, con inclusión de la banda de confianza al nivel de significación $90 \%(\mathrm{~b} 1, \mathrm{~b} 2)$. D. Estación meteorológica de Jaca, con inclusión de la misma banda de confianza para una serie teórica de 100 años (c1, c2) 


\section{Variabilidad espacial del riesgo de precipitaciones extremas y de la incertidumbre}

En la Figura 3 se ha representado en forma de mapa el valor de la precipitación de 100 años de retorno $\left(\mathrm{P}_{100}\right)$, junto con la banda de confianza al nivel de significación $90 \%$. El valor de $\mathrm{P}_{100}$ puede interpretarse como un estimador del riesgo de precipitaciones extremas en un lugar, y se considera un importante umbral para la activación de procesos geomorfológicos de envergadura como erosión lateral en cauces, removilización general de

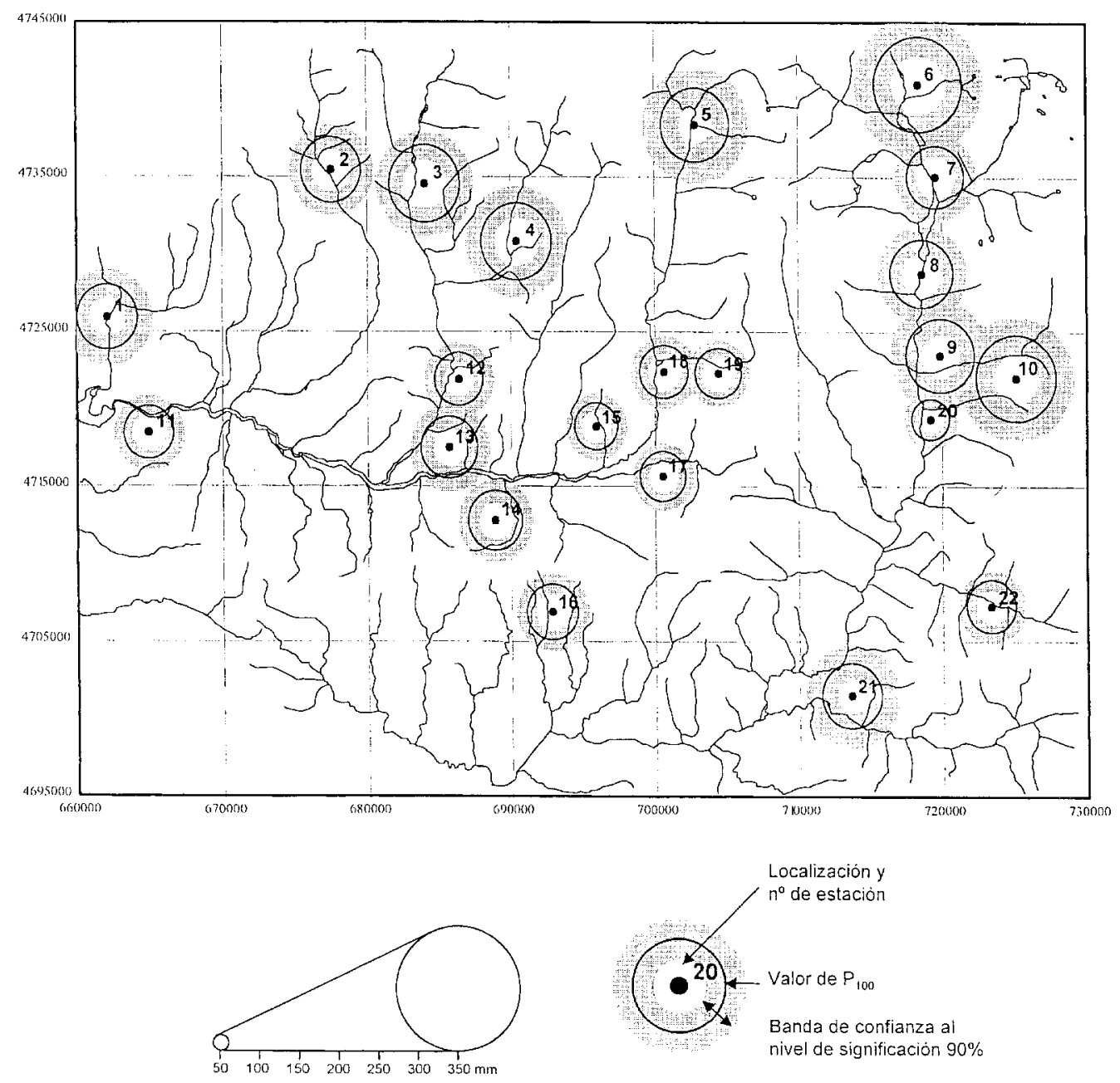

Figura 3. Distribución de P100 (precipitación máxima en 24 horas para un período de retorno de 100 años) en las estaciones analizadas. La orla gris representa la banda de confianza al nivel de significación $90 \%$. Clave de las estaciones en la figura 1 
conos de deyección o reactivación de grandes movimientos en masa (GARCÍA RUIZ et al., en prensa $a$; GARCÍA RUIZ et al., en prensa $b$ ). El examen de la figura revela una clara diferencia entre las estaciones de la Depresión Media y la mayor parte de la unidad del flysch, por un lado, y las del alto Pirineo y la zona más septentrional del flysch, por otro. Las estaciones del primer grupo presentan en general valores de $\mathrm{P}_{100}$ menos elevados, lo que significa un menor riesgo de precipitaciones extremas. En la Tabla 1 se aprecia cómo el registro confirma al modelo, pues las mayores intensidades en $24 \mathrm{~h}$. se han registrado en las estaciones del segundo grupo, con numerosos casos en los que se superan los $100 \mathrm{~mm}$.

Se puede decir, pues, que la distribución espacial de las precipitaciones extremas sigue a grandes rasgos la de las precipitaciones medias anuales, con los máximos valores en el alto Pirineo e inmediatamente al sur de las Sierras Interiores. Se trata de la zona de máximas altitudes y energía de relieve, que favorecen en general la ascensión de las masas de aire y la formación de células convectivas. En las sierras del flysch y sobre todo en la Depresión Media el relieve es más moderado, lo que parece determinante en la menor ocurrencia de precipitaciones extremas.

CREUS y PUIGDEFÁBREGAS (1978) indican además la existencia de tres regímenes diferenciados en la distribución anual de las precipitaciones máximas. Al sur de las Sierras Interiores la máxima probabilidad de ocurrencia de precipitaciones máximas corresponde a los meses estivales, con máximo secundario otoñal, mostrando la importancia de las tormentas convectivas veraniegas y las perturbaciones otoñales. En la zona alta pirenaica se diferencian dos subregiones: la central-oriental, donde las precipitaciones máximas muestran un máximo netamente otoñal, y la occidental (valles de Hecho, Ansó y Roncal), donde los máximos invernales reflejan la mayor influencia oceánica.

La amplitud de la banda de confianza (Tabla 1) da una idea de la precisión de la estimación. Por ejemplo, para la estación de La Sarra (Sallent de Gállego) la precipitación calculada para 100 años de retorno es de $210 \mathrm{~mm}$, aunque a un nivel de significación $90 \%$ debería decirse que el valor se encuentra entre 98 y $323 \mathrm{~mm}$. Esto relativiza mucho la validez de las estimaciones de períodos de retorno, al menos en ciertos lugares y con los registros existentes. Una observación interesante es que la amplitud de la banda de confianza está muy bien correlacionada con $\mathrm{P}_{100}(r=0,93)$; es decir, cuanto mayor es el riesgo de lluvias extremas menos fiables son las estimaciones. En general, para la zona de la Depresión las estimaciones tienen más precisión que para el alto Pirineo. En Jaca, por ejemplo, el valor de $\mathrm{P}_{100}$ estaría entre 109 y $157 \mathrm{~mm}$, un margen mucho más razonable de cara a trabajos aplicados.

La amplitud de la banda de confianza (la imprecisión de la estimación) depende básicamente de dos parámetros: el tamaño de la muestra (número de años de registro), como se ha visto en la Figura 2D, y la varianza de la misma (irregularidad interanual de las precipitaciones máximas). Es decir, cuanta más información de partida se posea, y cuanto más homogénea sea la muestra, más fiable será la estimación. En el caso de las estaciones del Pirineo la longitud de las series no ha sido el factor de más peso en el control del error, con- 


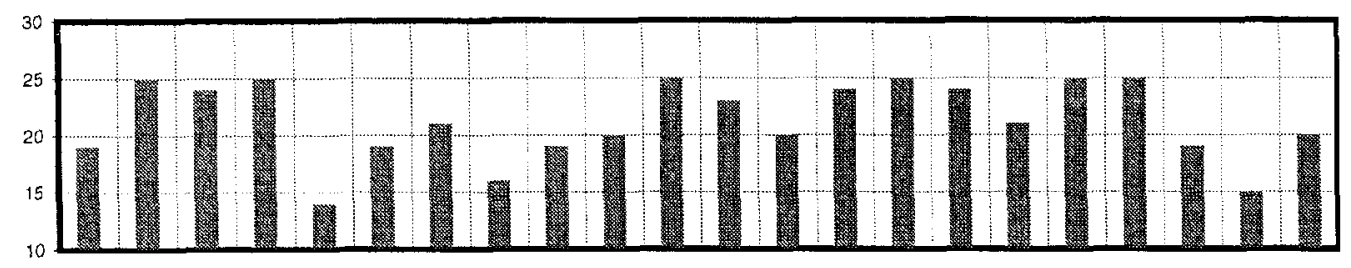

\section{Años de observación}

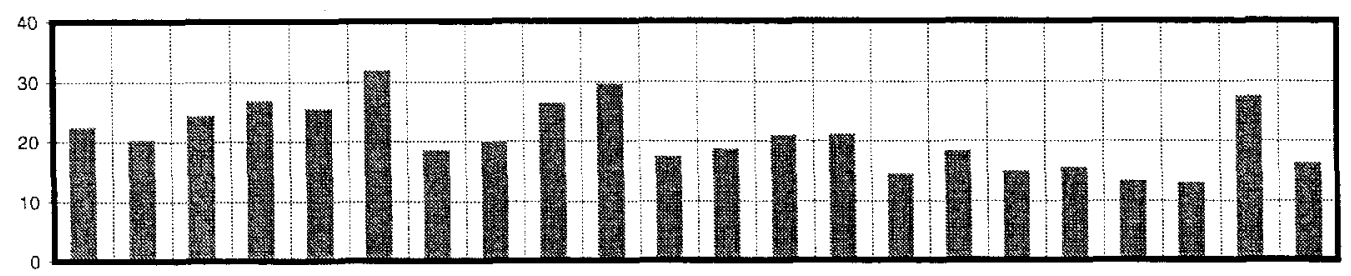

\section{Desviación típica de la serie de lluvias máximas $\left(\mathrm{mm} \mathrm{m}^{-2}\right)$}

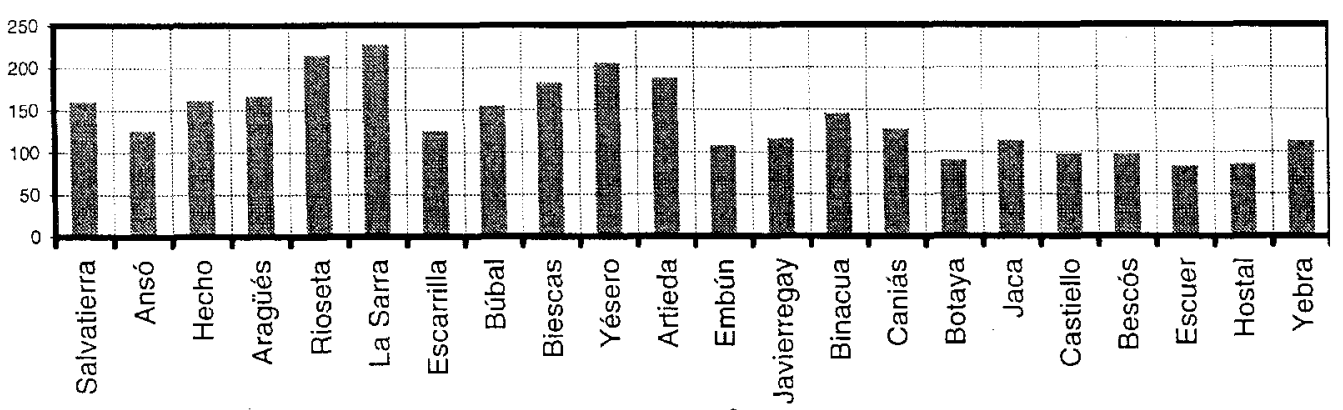

\section{Amplitud de la banda de confianza $\left(\mathrm{mm} \mathrm{m}^{-2}\right)$}

Figura 4. Relación entre la amplitud de la banda de confianza y diversos parámetros

trariamente a lo que cabría esperar. En cambio, la irregularidad de las series de precipitaciones máximas presenta una buena correlación con la amplitud de la banda de confianza $(r=0,7)$. En general las estaciones del alto Pirineo presentan una mayor irregularidad interanual que las estaciones de la Depresión, y esta es la principal razón de que estas últimas den lugar a estimaciones más precisas. En la Figura 4 se comparan estos tres valores para todas las estaciones utilizadas. El examen visual permite comprobar la elevada correlación entre la desviación típica de las lluvias máximas y la amplitud de la banda de confianza. 


\section{Cartografìa del riesgo de precipitaciones extremas mediante técnicas de regresión múltiple}

El cálculo de períodos de retorno en una red relativamente densa de estaciones climatológicas permite realizar estudios de distribución espacial del fenómeno, como se ha demostrado. A continuación se ha querido extender esta información desde los puntos de toma de datos a la totalidad del territorio de estudio, para disponer de una cartografía continua. Algunos procedimientos habituales son el uso de algoritmos de interpolación espacial como el krigging o las superficies de splines o, más adecuado al caso que nos ocupa, el empleo de modelos estadísticos de correlación con otra variable conocida, como la altitud. En otros trabajos se ha aplicado esta última técnica a las precipitaciones máximas, tropezando con importantes dificultades debido al propio carácter infrecuente y errático del fenómeno. En concreto, GARCÍA RUIZ et al. (1998) encontraron escasas correlaciones entre precipitaciones máximas y diferentes variables topográficas, encontrando ciertos patrones espaciales sólo para las precipitaciones más moderadas entre las extremas. Dado el interés práctico del estudio espacial de riesgos ambientales, se ha querido profundizar en esta metodología para poder llegar a ofrecer una cartografía del riesgo de precipitaciones extremas.

La Tabla 2 muestra los coeficientes de correlación lineal entre la precipitación media anual y $\mathrm{P}_{100}$ y las variables topográficas y de posición que se han comentado anteriormente. Las correlaciones entre la precipitación media anual y las variables regresoras son bastante buenas y permiten la utilización de modelos de interpolación espacial, sobre todo si se considera cada valle por separado (GARCÍA RUIZ et al., 1985). Sin embargo, al tratar con valores extremos $\left(\mathrm{P}_{100}\right)$ los coeficientes de correlación empeoran, sin llegar alcanzar el 0,60 , confirmando los pobres resultados obtenidos en otros estudios (WHITE y GARCÍA RUIZ, 1998; GARCÍA RUIZ et al., en prensa $a$ ).

\begin{tabular}{|lcc|}
\hline & Precipitación media anual & $P_{100}$ \\
\hline $\mathrm{P}_{100}$ &, 47 & - \\
Banda de confianza de $\mathrm{P}_{100}$ &, 32 &, 93 \\
Precipitación anual (promedio) & - &, 47 \\
Precipitación anual (desviación) &, 52 &, 22 \\
Easting &, 39 &, 34 \\
Northing &, 82 &, 26 \\
Elevación &, 70 &, 59 \\
Desv 1 km &, 70 &, 58 \\
Max 1 km &, 80 &, 58 \\
Min 1 km &, 80 &, 55 \\
Grad 1 km &, 68 &, 53 \\
\hline
\end{tabular}

Tabla 2. Coeficientes de correlación lineal 
El análisis de regresión múltiple resultó mucho más satisfactorio que la correlación de variables una a una. El modelo de nueve parámetros seleccionado como óptimo explica el $86 \%$ de la variabilidad de $\mathrm{P}_{100}$. Los parámetros son estadísticamente significativos, al presentar valores de $\mathrm{P}$ inferiores al valor crítico de 0,1 (nivel de significación 90\%). El error estándar de los valores estimados por el modelo sobre los observados es de $14,4 \mathrm{~mm}$, y los residuales no muestran estructura. Los parámetros del modelo son: coordenadas UTM; elevación; desv. $1 \mathrm{Km}, 3 \mathrm{Km}, 5 \mathrm{Km}$ y $8 \mathrm{Km}$; grad. 3 . Km y 5.Km. Estas variables están relacionadas con: I) la heterogeneidad del relieve, II) el gradiente topográfico y III) la localización geográfica, que controla la influencia atlántica/ mediterránea.

La conversión del modelo de regresión múltiple en un modelo espacial no resulta complicada con la ayuda de un SIG matricial, siempre que se disponga de coberturas continuas de las variables regresoras. En este trabajo se ha utilizado el software IDRISI para las operaciones principales, y el módulo GRID de ARC-INFO para obtener algunas de las capas de información. Al tratarse de variables topográficas, todas las capas se derivaron de un modelo digital de elevaciones (MDE). La ecuación paramétrica seleccionada como óptima por el paquete estadístico se aplicó pixel a pixel a toda la zona de estudio. El resultado se puede ver en la Figura 5. La distribución del riesgo de precipitaciones extremas según este mapa se ajusta bien al patrón espacial expuesto más arriba.

No hay que olvidar, sin embargo, que al hablar de $P_{100}$ nos referimos a una variable estimada con notable indeterminación. La buena correlación existente entre $\mathrm{P}_{100} \mathrm{Y}$ la amplitud de su banda de confianza $(r=0,93)$ ha permitido incluir esta indeterminación en la leyenda del mapa de riesgos, mediante una simple regresión lineal. La imprecisión aumenta considerablemente en los valores superiores de la leyenda.

Por último hay que tomar en consideración el hecho de que las estaciones pluviométricas existentes no son representativas de todo el territorio estudiado: existen áreas más altas, con mayor pendiente, o gradiente topográfico, etc., que cualquiera de ellas. En todas estas áreas el modelo realiza una extrapolación, y los resultados hay que tomarlos con mayor precaución. La Figura 6 muestra las zonas fuera del rango de la información inicial (zonas de extrapolación). Se observa que estas zonas han obtenido valores de $\mathrm{P}_{100}$ bastante superiores a los calculados para cualquiera de las estaciones.

\section{CONCLUSIONES}

La metodología de cálculo de períodos de retorno de lluvias extremas presenta graves problemas de tipo práctico, debido a la deficiencia de los registros pluviométricos disponibles y al propio hecho de trabajar con los valores extremos de la distribución. Es imprescindible incluir las bandas de confianza al calcular los períodos de retorno para cualquier 


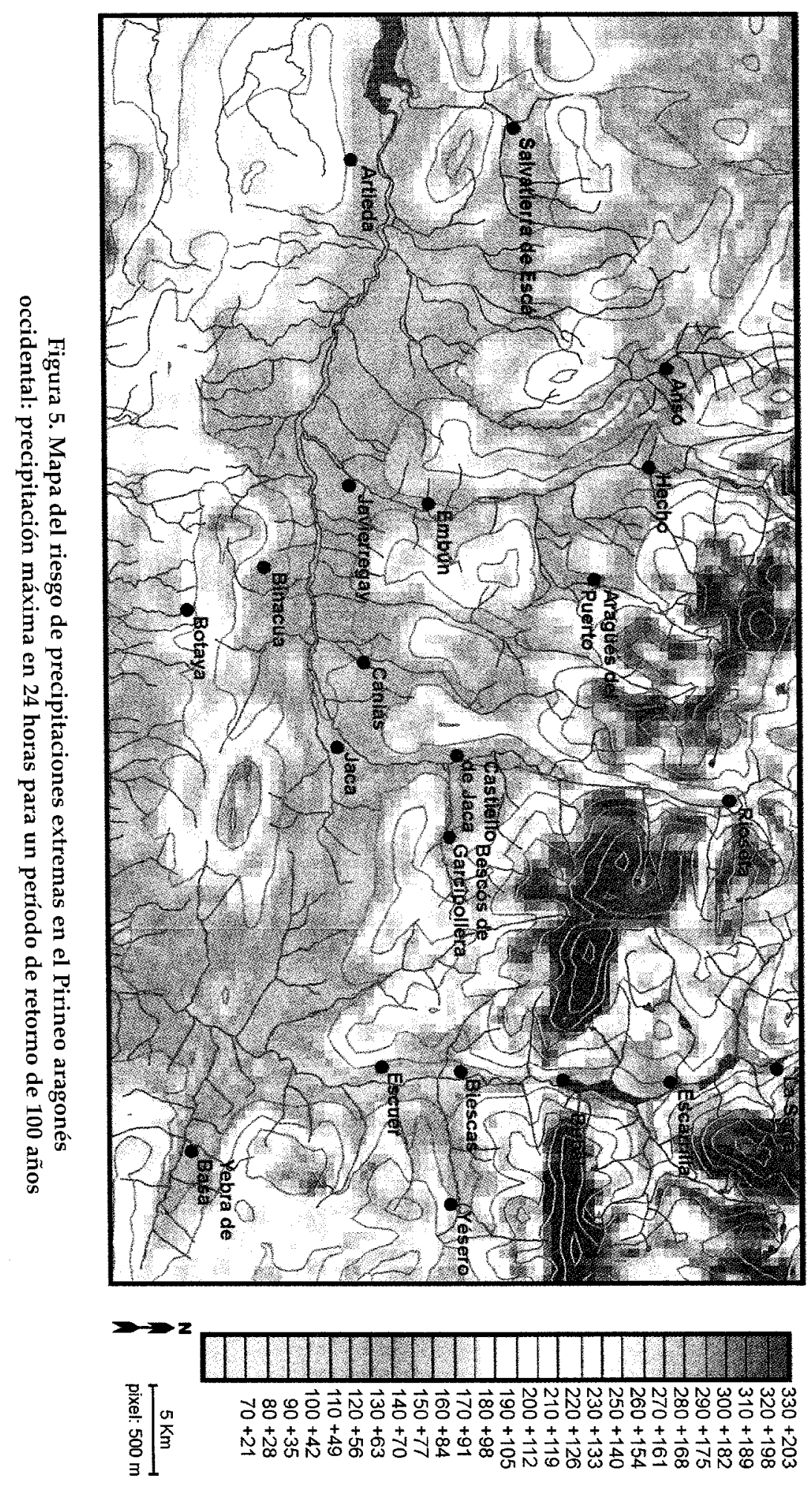




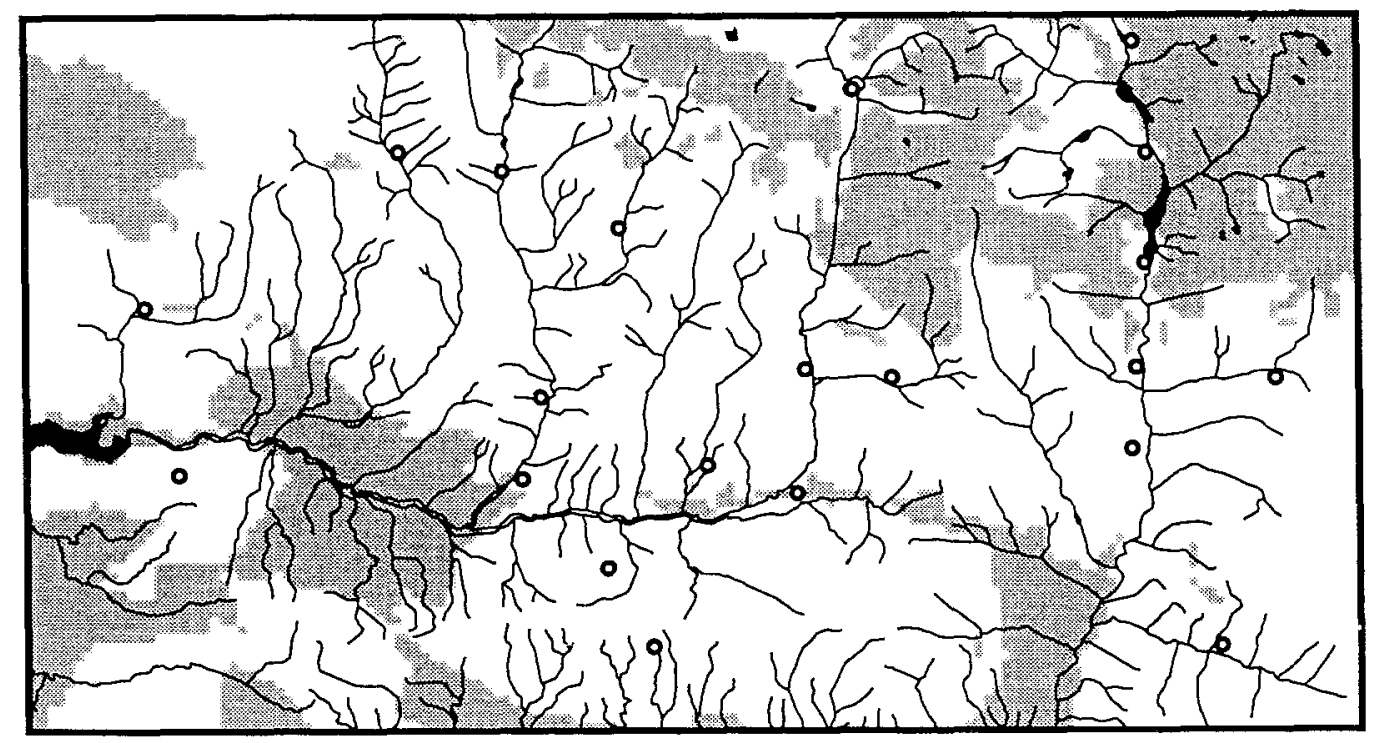

Figura 6. Zonas de extrapolación (fuera del rango de variación de los parámetros de la regresión)

trabajo, pues nos dan una idea de la precisión de los parámetros estimados y de la validez de los datos iniciales. En gran parte del Pirineo al menos, el margen de error de las estimaciones de períodos de retorno es tan grande que éstas tienen una utilidad muy relativa. Sólo en zonas con precipitaciones bastante regulares (raro en nuestro ámbito de estudio) o allí donde se cuente con registros climatológicos prolongados las estimaciones de períodos de retorno son razonablemente fiables.

Hay que tener en cuenta que el error de la estimación crece geométricamente conforme aumentamos el plazo de la predicción. En los estudios de riesgos para infraestructuras y actividades económicas posiblemente sea mejor considerar el límite superior de la banda de $\mathrm{P}_{100}$ que tomar como buena la precipitación de 200 o 500 años de retorno. La pérdida en precisión queda compensada con una ganancia en confianza, de la que se carece si no se realiza el estudio de significación. También parece clara la conveniencia, en estos casos, de abordar un estudio regional, en lugar de trabajar sólo con los datos de la estación más próxima a la ubicación de la obra que se proyecta.

Considerado globalmente, el riesgo de precipitaciones extremas en el Pirineo es elevado, como sabemos por otra parte por el registro histórico de catástrofes. El estudio de los datos registrados en las diferentes estaciones climatológicas permite afirmar la existencia de un patrón en la distribución de las precipitaciones extremas en la zona de estudio. Los valores más elevados se dan en el alto Pirineo e inmediatamente al sur de las Sierras Interiores, 
calculándose eventos máximos de 140 a $240 \mathrm{~mm}$ en 24 horas para un retorno de 100 años. En el resto de las sierras del flysch y en la Depresión Media se calculan valores máximos entre 80 y $140 \mathrm{~mm}$ en 24 horas para la misma recurrencia. Esta distribución parece confirmar la influencia del relieve local en la distribución de las precipitaciones extremas.

En cuanto a la elaboración de una cartografía del riesgo de lluvias extremas en el Pirineo aragonés, la metodología de regresión múltiple ha resultado bastante satisfactoria, permitiendo llegar a un modelo que minimiza los residuales. El mapa resultante adolece de una notable imprecisión derivada de la de los datos de partida, aunque se ajusta bien a la distribución de las lluvias máximas indicada anteriormente.

\section{AGRADECIMIENTOS}

Este trabajo se ha elaborado con el apoyo del proyecto de investigación «Water Resources Management In a Changing Environment: The impact of sediment on sustainability» (WARMICE-ENV4-CT98-0789) y «Debris fall assessment in mountain catchments for local end-users» (DAMOCLES-EVG1-1999-00027P), financiados ambos por la Comisión Europea, y «Estaciones permanentes para el estudio de procesos hidrológicos en ambientes mediterráneos» (EPROHIDRO: HID98-1056-C02-01) y «Producción de sedimentos y escorrentía como consecuencia de los cambios de uso del suelo en áreas de montaña» (PROSECUS: AMB96-0401), financiados por la CICYT.

\section{BIBLIOGRAFÍA}

APARICIO, F. J. (1997): Fundamentos de Hidrologin de superficie. Limusa-Noriega editores, 303 p., México.

BRU, J., SERRAT, D. y VILAPLANA, J.M. (1984): «La dinámica geomorfológica de la cuenca del torrente de Jou-La Guingueta (Noguera Pallaresa)». En Inestabilidad de laderns en el Pirineo, ETSI de Caminos, Canales y Puertos, 1.2.1-1.2.10, Barcelona.

CHOW, V.T., MAIDMENT, D.R. y MAYS, L.W. (1994): Hidrologín aplicadn. McGraw-Hill, 584 p., Santafé de Bogotá (1ª ed. ing, 1988).

CLOTET, N., GARCÍA RUIZ, J.M. y GALLART, F. (1989): «High magnitude geomorphic work in Pyrenees range: unusual rainfall event, November 1982». Studia Geomorphologica CarpathoBalcanica, 23, 69-92.

CREUS NOVAU, J. (1983): El clima del Alto Aragón occidental. Instituto de Estudios Pirenaicos, Jaca. «Monografías del Instituto de Estudios Pirenaicos», 109. 
CREUS NOVAU, J. y PUIGDEFÁBREGAS, T. (1978): «Influencia del relieve en la distribución de las precipitaciones máximas: un ejemplo pirenaico». Cuadernos de Investigación (Geografine Historin), $4(1), 11-23$.

CUSTODIO, E. y LLAMAS, M.R. (1996): Hidrologín subterrinea, vol. I. Barcelona, 1.157 p., Omega (1 ed. 1984).

DUNNE, T. y LEOPOLD, L.B. (1978): Water in environmental planning. Freeman \& Co., 818 p., San Francisco.

GARCÍA RUIZ, J.M., MARTÍ-BONO, C., LORENTE, A. y BEGUERÍA, S. (en prensa a): «Geomorphological consequencies of frequent and infrequent pluviometric and hydrological events in a mediterranean mountain area».

GARCÍA RUIZ, J.M., ARNÁEZ, J., WHITE, S. M., LORENTE, A. y BEGUERÍA, S. (en prensa b): «Uncertainty assessment in the prediction of extreme rainfall events: An example from the Central Spanish Pyrenees». Hydrological Processes.

GARCÍA RUIZ, J.M., BEGUERÍA, S. y LORENTE, A. (1999): «Eventos hidrológicos de baja frecuencia en el Pirineo central español y sus efectos geomorfológicos». Serie Geográfica.

GARCÍA RUIZ, J.M., ARNÁEZ, J., WHITE, S.M. y BORDONABA, A.P. (1998): «Predicción de eventos extremos en áreas de montaña. El ejemplo del Pirineo Central». En A. Gómez Ortiz y F. Salvador Franch (eds.), Investigaciones recientes de la Geomorfología española, Barcelona, p. 623-630.

GARCÍA RUIZ, J.M., WHITE, S.M., MARTÍ BONO, C., VALERO, B., ERREA, M.P. y GÓMEZ VILLAR, A. (1996): La catástrofe del barranco de Arás (Biescas, Pirineo arngonés) y su contexto espaciotemporal. Instituto Pirenaico de Ecología, 54 p., Zaragoza.

GARCÍA RUIZ, J.M., PUIGDEFÁBREGAS, J. y CREUS, J. (1985): Recursos hidricos superficinles del Alto Aragón. Instituto de Estudios Altoaragoneses, Huesca.

GUMBEL, E. J. (1967): Statistics of Extremes. New York, Columbia Univ. Press.

JONES, J. A. (1997): Global Hydrology. Processes, resources and environmental manngement. Harlow, 399 p., Longman.

LLAMAS, I. (1993): Hidrologín general. Principios y aplicnciones. Universidad del País Vasco, 635 p., Bilbao.

MARTÍ BONO, C.E. y PUIGDEFÁBREGAS, J. (1983): «Consecuencias geomorfológicas de las lluvias de noviembre de 1982 en las cabeceras de algunos valles pirenaicos». Estudios Geográficos, 170$171,275-290$. 
MARTÍN VIDE, I. (1985): «Estacionalidad de la precipitación y mediterraneidad en el Pirineo catalán». Notes de Geografía Física, 13-14,57-65.

VILAR, F. (1995): «Situaciones, riesgo y recurrencia de tormentas intensas en el Pirineo oriental». En J. Creus (ed.), Situaciones de riesgo clintático en España, Instituto Pirenaico de Ecología, 105116, Jaca.

WHITE, S. M. y GARCÍA RUIZ, J. M. (1998): «Extreme Erosional Events and their role in Mountain Areas of Northern Spain». Ambio, 27(4), 300-305.

WHITE, S., GARCÍA RUIZ, J.M., MARTÍ BONO, C., VALERO, B., ERREA, M.P. y GÓMEZ VILLAR, A. (1997): «The Biescas campsite disaster and its temporal and spatial context». Hydrological Processes, 11, 1794-1812. 